\title{
A Three-Dimensional Finite Element Model Constructed Using Magnetic Resonance Images of a Knee
}

\author{
Sheng-Liang $\mathrm{ZHANG}^{1}$, Jing XIA ${ }^{1}$, Tong-Tong GUO ${ }^{1, *}$ \\ ${ }^{1}$ Nature Science Department, Harbin Institute of Technology Shenzhen Graduate School, \\ 518000Shenzhen, China \\ tongtong.guo@hitsz.edu.cn
}

Keywords: Biomechanics, Knee Joint, Non-Linear, Finite Element Model.

\begin{abstract}
In recent years, MRI studies have resulted in a better understanding of the movement and deformation of the meniscus in the terbinafine connection and provide, information on the morphology of the articular cartilage. In this paper, we will use of computer-aided technique and MRI to reconstruct the shape of the living knee, and divide the mesh and add the material properties to create the three-dimensional nonlinear knee joint finite element model information. Examining the relative sliding and friction in the knee joint, I used the dynamic dominant non-linear finite element technique to simulate the photomechanical properties of the tibial femoral joint under different loads. Apply a pressure, in the 0 degree direction to calculate the change of each structure, including: maximum pressure, average pressure, contact pressure, internal stress.
\end{abstract}

\section{Dimensional Finite Element Model of Knee Joint was Established Based on MRI}

All of the data from this study were obtained from the 25-year-old healthy male knee joint. The equipment was selected from GE's MRI Signa product line and equipped with DICOM 3.0 international standard interfaces for image output of DICOM standard format.

\section{Simplware Software to Build STL Format Model}

We use image processing software Simpleware-ScanIPV3.1 to complete the entire area of the knee the three-dimensional reconstruction of the structure. Since the whole image needs to be divided into the patella, femur, tibia and their surface of the cartilage and medial and lateral meniscus, need to establish a number of different mask processing, and finally through the Boolean operation to remove the overlap between the different parts of the output data.
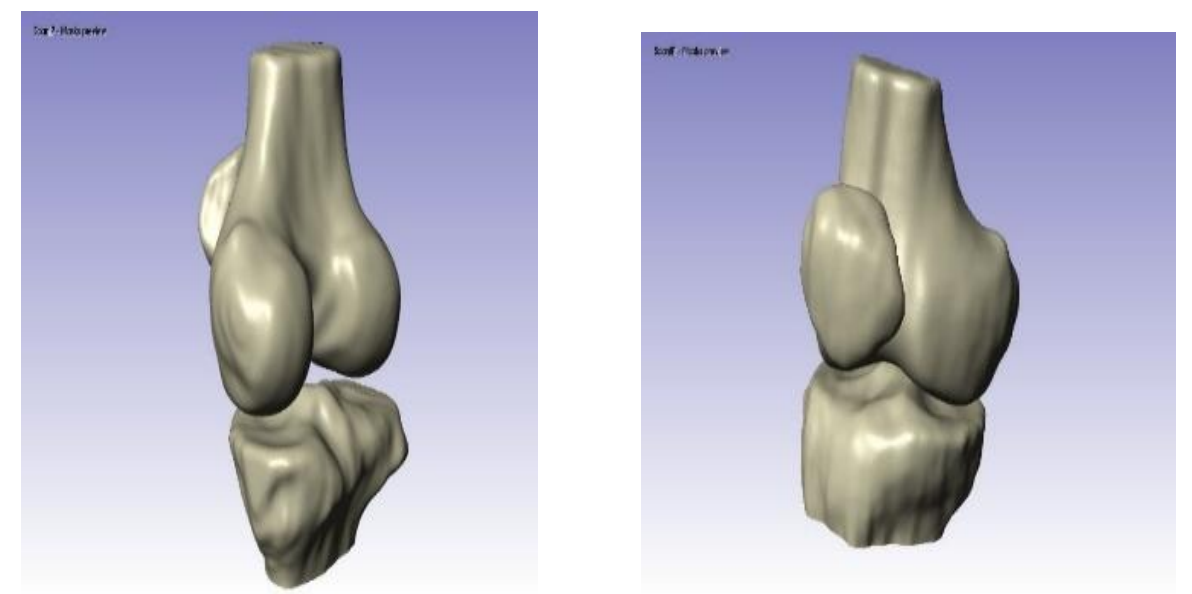

Figure 1-1. Digital image processing after three-dimensional reconstruction of the knee joint 

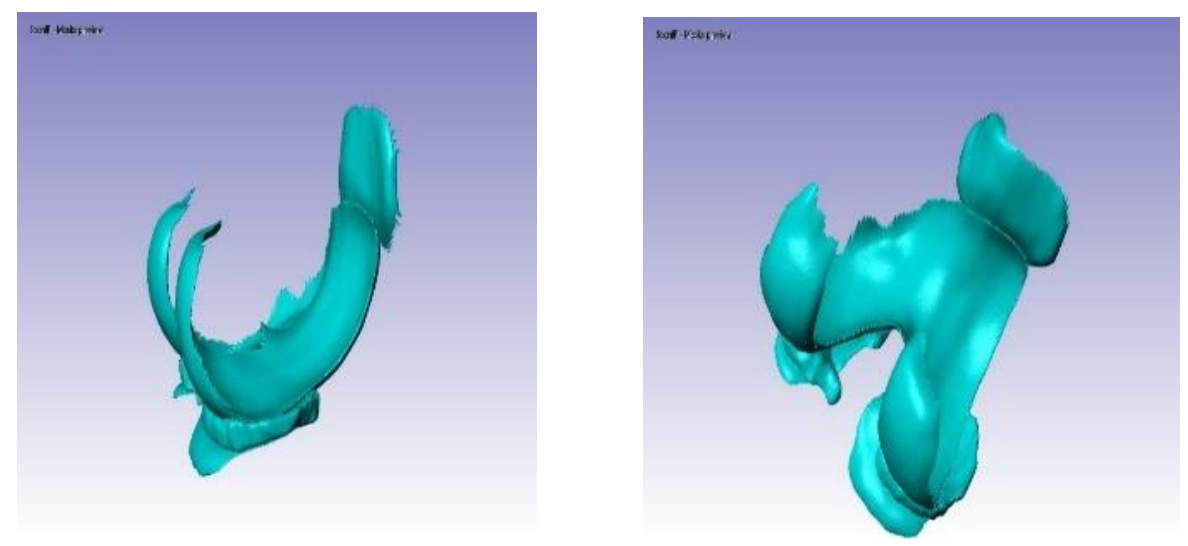

Figure 1-2. Digital image processing after three-dimensional reconstruction of the articular cartilage of the knee

\section{Partitioning Meshes in Mimics Software}

Figure 1-3 shows the procedure

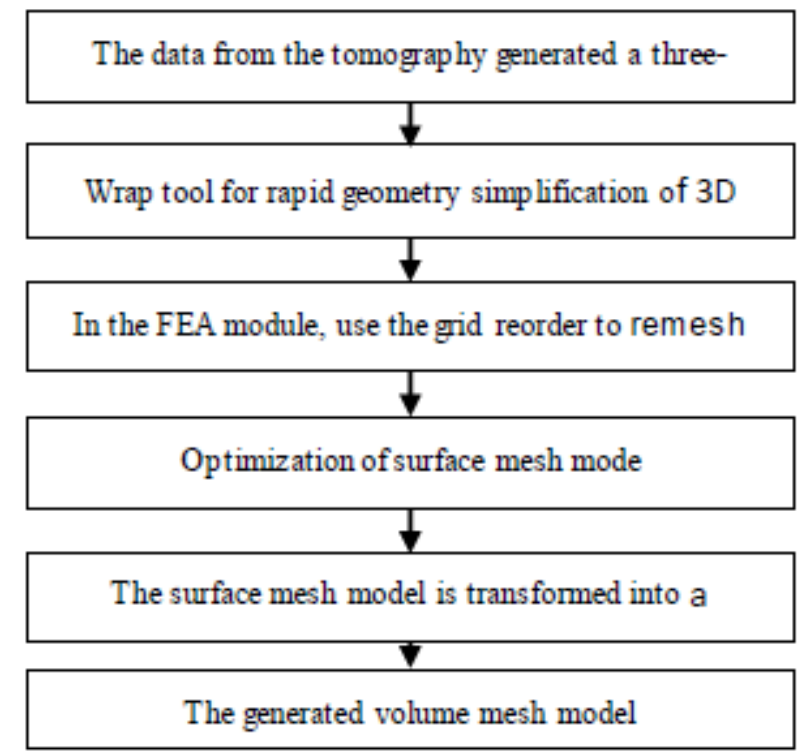

Figure 1-3. The procedure

The resulting knee joint model is shown in Figure 1-4

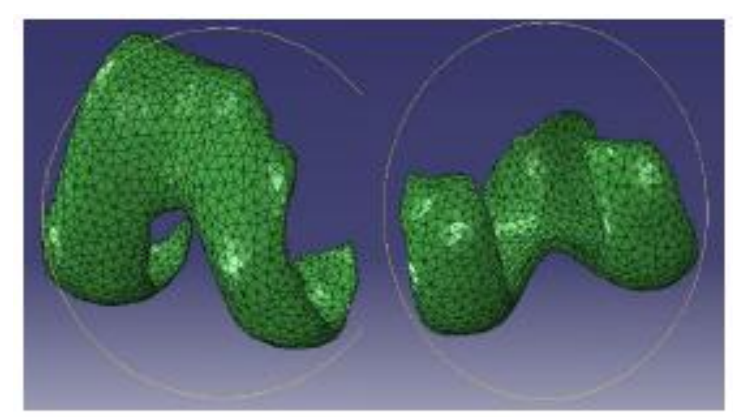

a) Frontal view of the femoral cartilage b) Rear view of the femoral cartilage

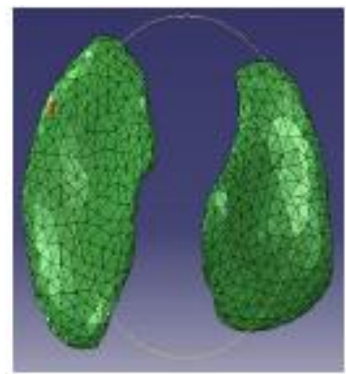

c) Tibia plateau cartilage

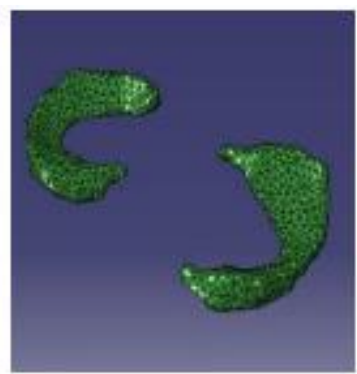

d) Medial and lateral meniscus

Figure 1-4. Finite element model of knee joint 


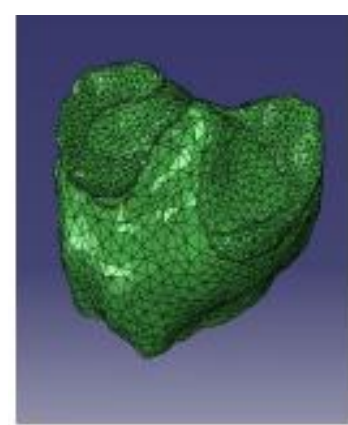

e) Tibia plateau

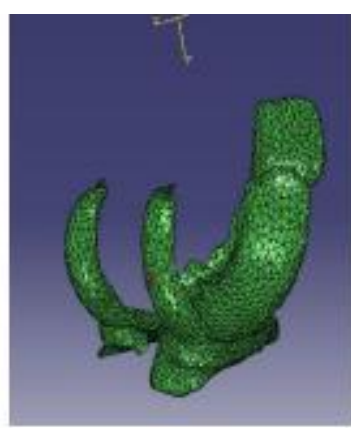

f) Knee joint cartilage

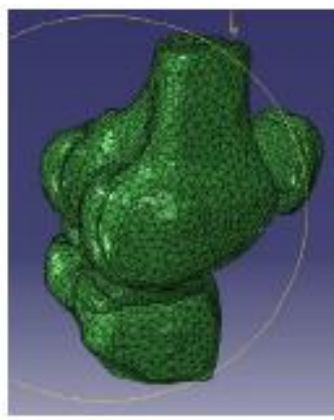

g) Knee side view

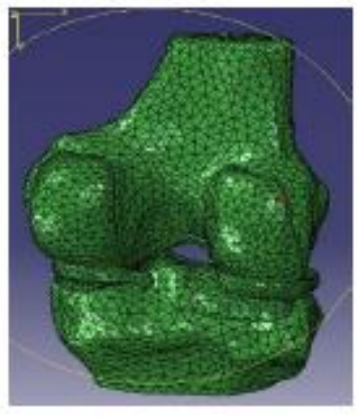

h) Rear view of the knee

Figure 1-4. Finite element model of knee joint

\section{The 3D Finite Element Model of the Knee Joint was established}

The ligaments of the anterior and posterior horn of the meniscus and the tibia plateau ligaments were simulated using linear springs. Taking into account the actual ligament width, 10 springs were used for each ligament approximation. The stiffness of the meniscus ligaments was $2000 \mathrm{~N} / \mathrm{mm}$, is $200 \mathrm{~N}$ $/ \mathrm{mm}$, the spring connection is shown in Figure 1-6.
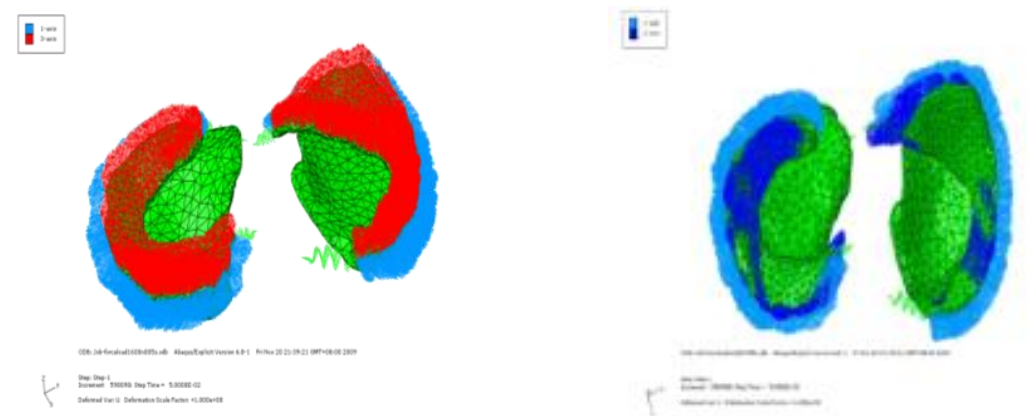

Figure 1-5. Direction of cross-direction and radially-oriented fiber bundles

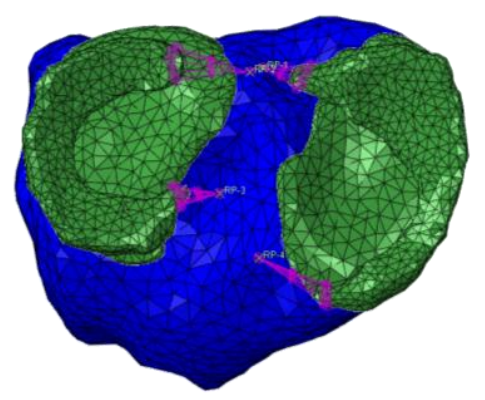

Figure 1-6. Meniscus anteroposterior angle and tibial plateau linear spring connection

\section{Boundary Conditions and Loads}

The weight of the study subjects was $70 \mathrm{~kg}$. When the feet were balanced, the weight of one knee was approximately equal to the value of $10 \mathrm{~cm}$ in the knee. When in the gait, the center of gravity of the load are all distributed in the unilateral knee, this time a single knee bearing. The direction is vertical downwards.

\section{Biomechanical Properties of Femoral Tibial Joint}

The finite element analysis of the tibiofemoral joint is shown in Figure 3-1 


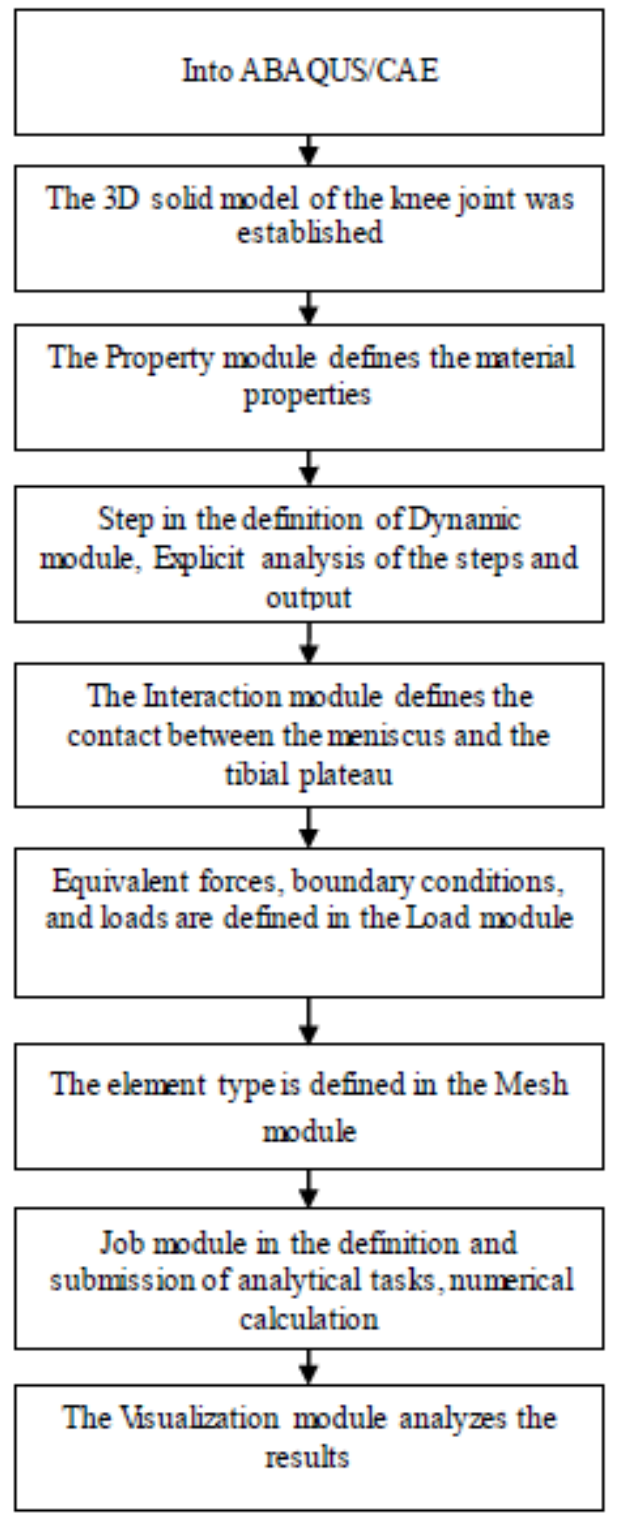

Figure 3-1. ABAQUS / Explicit Analysis Flowchart

\section{Tibiofemoral Joint Contact Characteristics}

In this study, loads of $600 \mathrm{~N}$ and $1600 \mathrm{~N}$ were loaded on the finite element model of tibia femur to simulate body weight and external load respectively.

Figures 3-2 show the contact pressure of the tibial plateau cartilage at $600 \mathrm{~N}$ and $1600 \mathrm{~N}$ loads, respectively

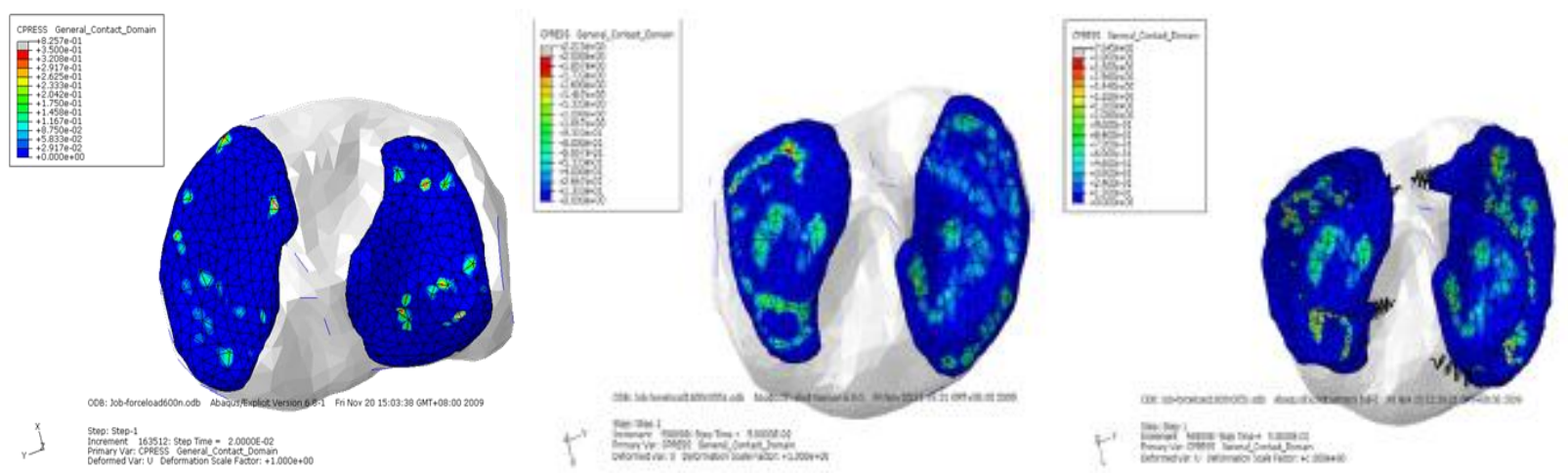

Figure 3-2. Contact pressure of the tibial plateau cartilage and meniscus at $1600 \mathrm{~N}$ load 


\section{Biomechanical Characteristics of Meniscus}

When the external load of $600 \mathrm{~N}$ and $1600 \mathrm{~N}$, the Mises meniscus stress distribution shown in Figure 3-3 to 3-4, medial and lateral meniscus Mises stress mainly in the anterior and posterior angle and the meniscus inner edge, meniscus The stress of the posterior horn is greater than that of the anterior horn, and the Mises stress increases with the increase of the load. And in the meniscus of the inner edge, abdomen, posterior horn near the corner and the stress is more concentrated. From the loss of clinical meniscus, the loss is mainly in the meniscus inner edge, abdomen and anteroposterior angle. The mechanical analysis of the meniscus injury is given.

Figure 3-5 for the tibiofemoral joint under different load meniscus in front and back, inside and outside and up and down the direction of the displacement deformation. Figure 3-6in the $600 \mathrm{~N}$ load on the medial meniscus overall backward movement, while the lateral half anterior horn to move forward, the rest of the backward movement. With the increase in load at $1600 \mathrm{~N}$, the medial meniscus forward angle to move forward the rest of the back, so that the opening of the medial meniscus, lateral meniscus forward angle, the rest of the back, but the displacement of the posterior horn is less than its back. Figure 3-7 for the meniscus lateral displacement, in $600 \mathrm{~N}$ displacement of the medial meniscus overall outward movement, anterior horn displacement is greater than the posterior angular displacement, lateral meniscus anterior horn outward and rearward inward. When the displacement is $1600 \mathrm{~N}$, the whole displacement trend does not change greatly, but the displacement increases obviously. Figure 3-8, in the upper and lower meniscus front corner down, rear part of the upward movement. So in general with the increase in load, the medial and lateral meniscus toward the direction away from the center of the tibial plateau displacement, openings, posterior horn open meniscus.

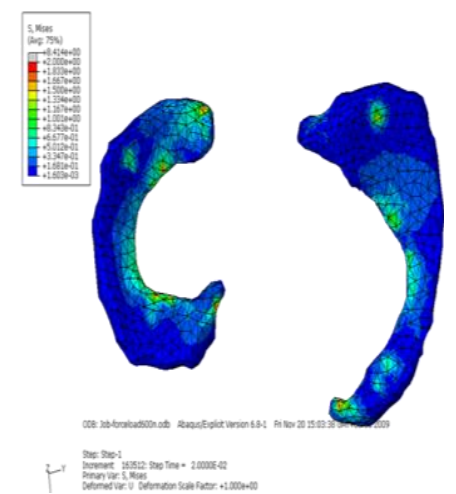

Figure 3-3. Mises Stress Moiré of meniscus under 600N load
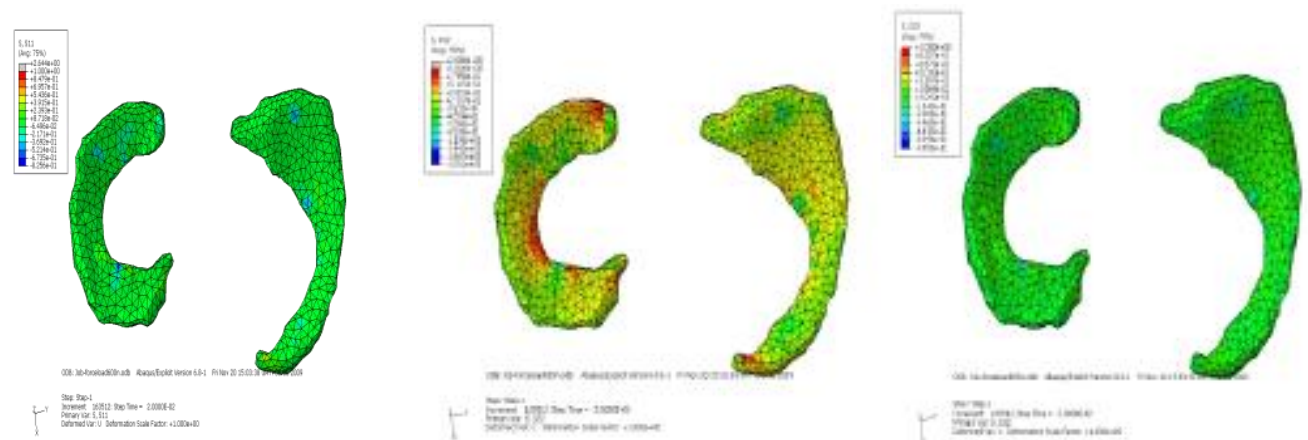

Figure 3-4. Normal stress moiré of meniscus along direction $1 \backslash 2 \backslash 3$ under $600 \mathrm{~N}$ load 

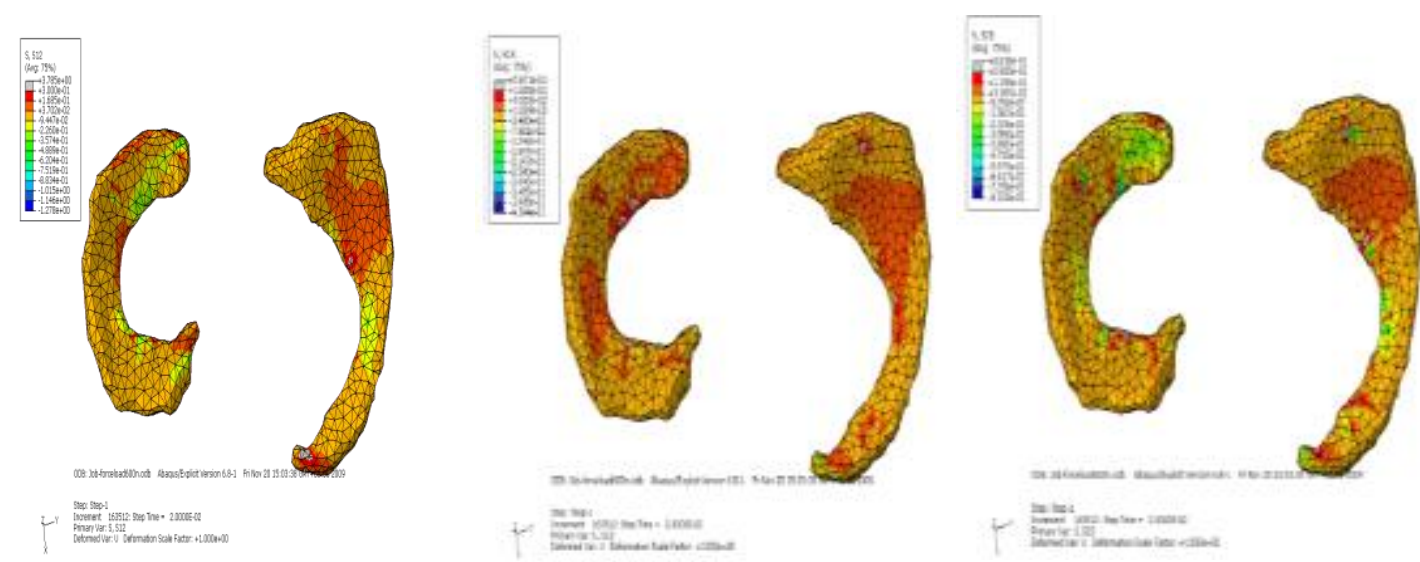

Figure 3-5. Shear stress pattern of meniscus along direction 1and $2 \backslash 1$ and $3 \backslash 2$ and3under $600 \mathrm{~N}$ load
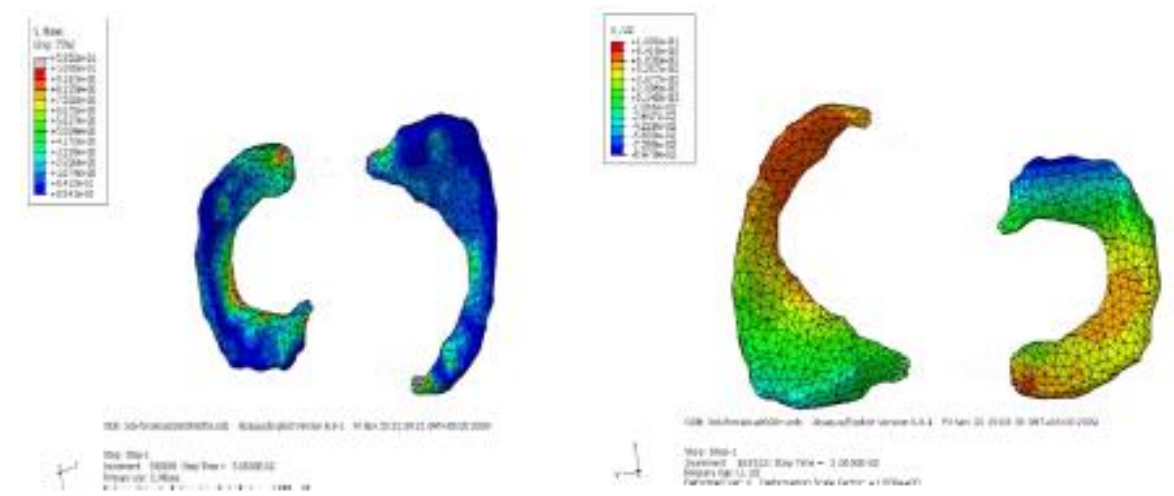

Figure 3-6. Mises Stress Moiré of meniscus under 1600 N and600N load
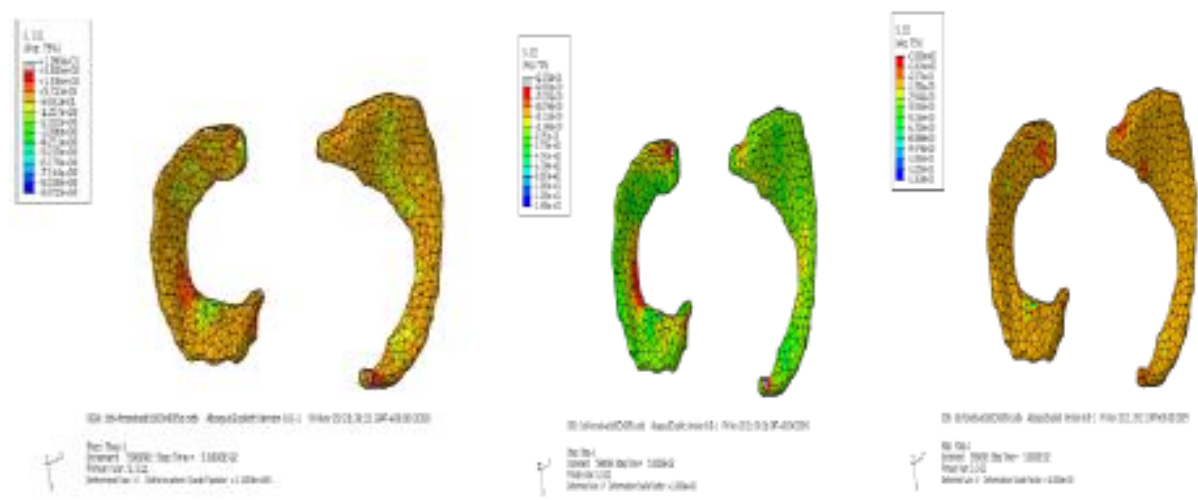

Figure 3-7. Normal Stress Moiré Pattern of the meniscus along direction $1 \backslash 2 \backslash 3$ under a $1600 \mathrm{~N}$ load
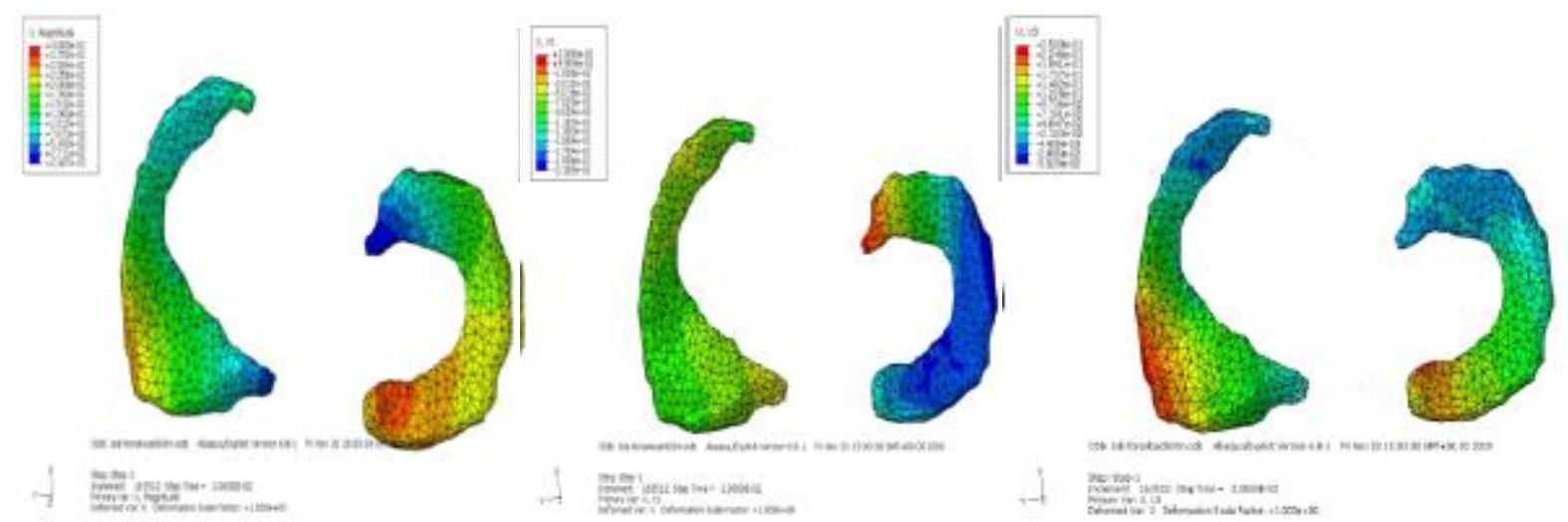

Figure 3-8.The overall displacement of the meniscus under $600 \mathrm{~N}$ load moiré 


\section{References}

1. M. H. Moeinzadeh, A. E. Engin, N. Akkas. Two-Dimensional Dynamic Modeling of Human Knee Joint. J.of Biomech. 16: 253-264(1983).

2. E. Abdel-Rahman, M. S. Hefzy. A Two Dimensional Dynamic Anatomical Model of the Human Knee Joint. ASME. J.Biomech.Eng. 115: 357-359(1993).

3. E. Abdel-Rahman, M. S. Hefzy. Three-Dimensional Dynamic Behavior of the Human Knee Joint under Impact Loading. Med. Eng. Phy. 20: 276-290(1988).

4. M. Z. Bendjaballah, A. Shirazi-Adl, D. J. Zukor. Biomechanics of the Human Knee Joint in Compression: Reconstruction, Mesh Generation and Finite Element Analysis. The Knee. 2(2):6979(1995).

5. G. Li, J. Gil. A Validated Three-Dimensional Computational Model of a Human Knee Joint. J.of Biomech.Eng. 121:657-662(1999). 